\title{
Collagen I-Matrigel Scaffolds for Enhanced Schwann Cell Survival and Control of Three-Dimensional Cell Morphology
}

\author{
Daniel D. Dewitt, M.S., ${ }^{1}$ Stephanie N. Kaszuba, M.S., ${ }^{1}$ Deanna M. Thompson, Ph.D., \\ and Jan P. Stegemann, Ph.D. ${ }^{1,2}$
}

We report on the ability to control three-dimensional Schwann cell (SC) morphology using collagen I-Matrigel composite scaffolds for neural engineering applications. SCs are supportive of nerve regeneration after injury, and it has recently been reported that SCs embedded in collagen I, a material frequently used in guidance channel studies, do not readily extend processes, instead adopting a spherical morphology indicative of little interaction with the matrix. We have modified collagen I matrices by adding Matrigel to make them more supportive of SCs and characterized these matrices and SC morphology in vitro. Incorporation of 10\%, 20\%, 35\%, and 50\% Matrigel by volume resulted in 2.4,3.5,3.7, and 4.2 times longer average SC process length after 14 days in culture than with collagen I-only controls. Additionally, only 35\% and 50\% Matrigel constructs were able to maintain SC number over 14 days, whereas an $88 \%$ decrease in cells from initial seeding density was observed in collagen-only constructs over the same time period. Mechanical testing revealed that the addition of $50 \%$ Matrigel increased matrix stiffness from $6.4 \mathrm{kPa}$ in collagen I-only constructs to $9.8 \mathrm{kPa}$. Furthermore, second harmonic generation imaging showed that the addition of Matrigel resulted in non-uniform distribution of collagen I, and scanning electron microscope imaging illustrated distinct differences in the fibrillar structure of the different constructs. Collectively, this work lays a foundation for developing scaffolding materials that are concurrently supportive of neurons and SCs for future neural engineering applications.

\section{Introduction}

$\mathrm{C}$ URRENT TREATMENTS for patients suffering from spinal cord injury rarely result in full functional recovery, necessitating the development of new therapeutic approaches. Schwann cells (SCs) are currently being investigated as a component of nerve repair strategies in the central nervous system (CNS) because of their close relationship with neurons in the peripheral nervous system (PNS) ${ }^{1-5}$ SCs play an important role in the PNS, and a number of studies have demonstrated the beneficial effect SCs have on nerve regeneration. $^{6-11}$

For these reasons, SCs have been used in CNS nerve repair strategies with promising results despite being resident cells of the PNS. In vitro, SCs and astrocyte substrata can support CNS neurite outgrowth. ${ }^{12}$ In vivo, SC-filled guidance channels implanted into transected rat spinal cords resulted in significantly more axon extension than with acellular controls. ${ }^{13,14}$ Entry into the distal nerve stump was reported in hemisected spinal cords when SC-loaded mini-channels were used. ${ }^{15}$ In some rare cases after injury, SCs will migrate into the CNS, where they can play a role in endogenous repair processes. ${ }^{16}$ In addition to promoting axonal outgrowth, autologous SCs can remyelinate CNS axons, which is necessary to restore function. ${ }^{17}$ Despite these successes, treatments for patients suffering from spinal cord injury remain inadequate, and full functional recovery has yet to be achieved. Incorporation of autologous SCs is a promising avenue to improve the outcome of these approaches.

Most efforts have focused on optimizing scaffolding environments solely for axonal extension, whereas concurrent optimization for SCs remains less studied. Creation of a scaffold optimized for SCs and neurons may yield better results. Hurtado et al. reported that few SCs implanted in fibrinfilled rat thoracic spinal cord grafts remained after 6 weeks, pointing out that SC survival needs to be improved to increase graft efficacy. ${ }^{18}$ Additionally, Rosner et al. recently reported that SCs embedded in three-dimensional (3D) collagen I, a material supportive of neurite outgrowth that is commonly used as a scaffold in guidance channel studies, exhibited a spherical morphology, indicative of little interaction with the matrix material. ${ }^{19}$ Although some studies have demonstrated

\footnotetext{
${ }^{1}$ Department of Biomedical Engineering, Rensselaer Polytechnic Institute, Troy, New York.

${ }^{2}$ Department of Biomedical Engineering, University of Michigan, Ann Arbor, Michigan.
} 
beneficial regenerative effects using SC-loaded collagen I hydrogels, none have specifically examined SC interaction with the matrix in the absence of complicating variables such as neurons, fibroblasts, proteins, and growth factors. ${ }^{3,20-22}$

It is likely that multiple cues present in the 3D scaffoldincluding glia, soluble factors, scaffold geometry and composition, and external forces-act synergistically to enhance neurite outgrowth. To systematically test these variables, a scaffolding material that is independently and concurrently supportive of SCs and neurons must be developed. Collagen I alone does not accomplish this goal but is a promising scaffold material because it is robust enough to be easily handled and can be directionally aligned. ${ }^{20,23}$ We therefore have augmented a collagen I matrix with Matrigel ${ }^{\mathrm{TM}}$, BD Biosciences (Franklin Lakes, NJ), a commercially available preparation of basement membrane proteins comprising approximately $60 \%$ laminin, 30\% collagen IV, and 7\% entactin. ${ }^{24}$ Matrigel was chosen because laminin and collagen IV are two prominent, endogenous basal lamina proteins of the nervous system that are in direct contact with SCs. ${ }^{25}$

Our goal in this work was to develop a scaffold material that is supportive of SC spreading to serve as a platform for investigating the synergistic contributions of multiple guidance cues on neurite outgrowth. We hypothesized that the addition of Matrigel to collagen I would support SC spreading-defined as the appearance of protrusions from the SC and a spindle-shaped morphology-and greater SC number because of changes in the chemistry and architecture of the scaffold. We used growth-factor-reduced (GFR) Matrigel to minimize the potential effects of soluble factors. Levels of insulinlike growth factor (IGF)-1 and transforming growth factor beta 1 (TGF- $\beta 1$ ) are not effectively reduced in the GFR formulation, and therefore we controlled for this by incorporating these proteins into pure collagen I matrices. In the present study, we have characterized collagen I-Matrigelcomposite scaffolds, as well as the effects of these materials on SC viability, number, and morphology. Scaffolds that are supportive of SCs and neurons will allow us to systematically investigate multiple guidance cues, with the ultimate aim of developing improved spinal cord injury.

\section{Materials and Methods}

\section{Cell culture}

Primary SCs were isolated from the sciatic nerves of neonatal rats and provided as a gift from Dr. J. Salzer (New York University, New York, NY). Subsequent expansion of SC cultures was carried out in Dulbecco's modified Eagle medium containing $4 \mathrm{mM}$ L-glutamine, $4.5 \mathrm{~g} / \mathrm{L}$ D-glucose, and $110 \mathrm{mg} / \mathrm{L}$ sodium pyruvate (DMEM; CellGro, Manassas, VA) and supplemented with $10 \%$ fetal bovine serum (FBS; HyClone, Logan, UT), $50 \mathrm{U}$ penicillin/streptomycin (Cellgro), $10 \mu \mathrm{g} / \mathrm{mL}$ bovine pituitary extract (Becton, Dickinson and Company, Franklin Lakes, NJ), and $10 \mu \mathrm{M}$ forskolin (SigmaAldrich, St. Louis, MO). Cells were grown at $37^{\circ} \mathrm{C}$ in $7 \%$ carbon dioxide, with the media being changed every other day. ${ }^{26}$ Cells were passaged at approximately $90 \%$ confluence using a $0.05 \%$ trypsin $/ 0.53 \mathrm{mM}$ ethylenediaminetetraacetic acid solution (CellGro), and cells between passages 10 and 15 were used for all experiments. Before use, SC purity was assessed in monolayer culture using S100 staining and quanti- fied using ImageJ (National Institutes of Health). Purity levels of greater than $95 \%$ were observed for all cultures from passages 10 to 15 .

\section{Construct preparation}

After trypsinization, SCs were diluted in growth medium, pelleted, and then resuspended in $10 \mathrm{~mL}$ of phenol-red free growth media (CellGro) to reduce background fluorescence; ${ }^{27}$ other than the absence of phenol red, this growth medium was identical to that described above. Cells were subsequently counted, partitioned, and pelleted again before use.

Each $0.5-\mathrm{mL}$ construct was seeded with 500,000 cells in 24well plates (BD Biosciences, San Jose, CA), resulting in an initial radius of $8 \mathrm{~mm}$ and a thickness of $2.5 \mathrm{~mm}$. Briefly, cells were pelleted and resuspended by subsequent additions of the following solutions: 5X DMEM (CellGro), phenol-red free growth medium, FBS, $0.1 \mathrm{~N}$ sodium hydroxide and $4 \mathrm{mg} / \mathrm{mL}$ of acid-solubilized bovine collagen type I stock (MP Biomedicals, Solon, $\mathrm{OH}$ ) at ratios of 2:1:1:1:5, respectively, resulting in a $2-\mathrm{mg} / \mathrm{mL}$ collagen solution with SCs. GFR Matrigel (BD Biosciences) was the final component incorporated-because it gels much more rapidly than the collagen-at $10 \%, 20 \%$, $35 \%$, or $50 \%$ by volume. Dilution with Matrigel at these percentages reduced the collagen I concentration to 1.8, 1.6, 1.3 , and $1.0 \mathrm{mg} / \mathrm{mL}$, respectively; constructs without any Matrigel retained the collagen I concentration of $2 \mathrm{mg} / \mathrm{mL}$. In some cases, the growth factors TGF- $\beta 1$ (R\&D Systems, Minneapolis, MN) or IGF-1 (Sigma) were incorporated at $2 \mathrm{ng} / \mathrm{mL}$ and $5 \mathrm{ng} / \mathrm{mL}$, respectively, in collagen-only gels as controls. Construct solutions were mixed using a micropipettor, transferred into well plates, and placed at $37^{\circ} \mathrm{C}$ for $30 \mathrm{~min}$ to cause gelation. Constructs remained fully hydrated during this time and subsequently were covered with $1 \mathrm{~mL}$ phenol red-free growth medium and loosened from the sides of the wells using a sterile plastic spatula. All constructs were cultured for 3,7 , or 14 days before analysis, with the medium being changed every 2 days. No compaction was observed in any of the constructs over 14 days in culture.

\section{Second harmonic generation and scanning electron microscope imaging}

For second harmonic generation (SHG) imaging, constructs were washed in phosphate buffered saline (PBS, Cambrex, Walkersville, MD) and fixed for $30 \mathrm{~min}$ at room temperature using a $4 \%$ paraformaldehyde (Sigma) $/ 4 \%$ sucrose (Sigma) solution. Samples were visualized using a laser scanning confocal microscope (Carl Zeiss, Thornwood, NY) equipped with a Ti-Sapphire femtosecond pulsed laser (Coherent, Inc. Santa Clara, CA) at $63 \times$ magnification. The laser was tuned to $820 \mathrm{~nm}$, and a bandpass filter of 390 to $465 \mathrm{~nm}$ was employed to visualize the collagen networks. ${ }^{28}$

Constructs were prepared for scanning electron microscopy (SEM) using an initial fixation in a $2 \%$ glutaraldehyde (Poly Scientific, Bay Shore, NY)/2\% paraformaldehyde solution for $1 \mathrm{~h}$. After a PBS wash, samples were exposed to serial dilution in increasing percentages of ethanol, up to $100 \%$. Samples were critically point dried using an Autosamdri-815 (Tousimis Research Corp., Rockville, MD) and sputter coated with platinum for visualization. All SEM imaging (Carl Zeiss 
SMT, Thornwood, NY) was performed on day 3 at a magnification of $25,000 \times$.

\section{Compliance testing}

Acellular composite constructs were prepared at $0 \%, 10 \%$, $20 \%, 35 \%$, and $50 \%$ Matrigel ( $n=3$ for each condition). The matrix bulk stiffness describes the mechanical properties the cells experience when embedded in the constructs. Unconfined compression testing was performed on day 3 using an EnduraTEC ELF 3200 system (Bose Corp., Eden Prairie, $\mathrm{MN}$ ) equipped with a $250-\mathrm{g}$ load cell and a $1.27-\mathrm{cm}^{2}$ Lexan platen. Constructs remained in medium at $37^{\circ} \mathrm{C}$ until testing commenced. The construct was brought up to the compression platen by eye and the load cell tared. Under displacement control, a constant strain rate of $0.5 \mathrm{~mm} / \mathrm{min}$ was applied, and the resulting force (in $\mathrm{N}$ ) was measured every $200 \mathrm{~ms}$. Stress-strain curves were created from these data. All stresses less than $0.4 \mathrm{kPa}$ were eliminated because they were assumed to be due to negligible interaction forces before the platen touched the surface of the gel. The strain was normalized to reflect this change. The linear region of the resulting curve was determined to be between $15 \%$ and $50 \%$ of the maximal strain. The slope of this linear region was calculated and the resulting elastic moduli graphed.

\section{Cell viability and number}

Cell viability and cell number in each construct were quantified after 3, 7, and 14 days in culture. Viability was analyzed using a vital dye kit (Molecular Probes, Carlsbad, CA). Constructs were washed in PBS then stained concurrently with calcein (1:1000) and ethidium homodimer $(\mathrm{EH}$, 1:500) diluted in PBS for 45 min followed by two PBS washes to remove excess dye. Live (calcein + ) and dead $(\mathrm{EH}+)$ cells were visualized using a laser scanning confocal microscope (Carl Zeiss) equipped with 488-nm and 543-nm lasers and a $10 \times$ objective. One hundred fifty- $\mu \mathrm{m}$ z-stacks were taken of each sample and subsequently analyzed using FARSIGHT (courtesy of Dr. Badri Roysam, Rensselaer Polytechnic Institute) software, which can segment and count cells. ${ }^{29}$ Z-stacks were taken of both sides of the constructs, and the location of image stacks was the same for all construct conditions to control for possible differences in diffusion characteristics. Total numbers of live cells and dead cells were tabulated for each construct type and output as a percentage of living cells (living cells/total cells). Three separate trials were performed, testing one of each construct type per trial.

Cell number was quantified using the CyQuant GR Cell Proliferation Kit (Molecular Probes). Whole constructs ( $\mathrm{n}=4$ per construct type) were frozen at $-80^{\circ} \mathrm{C}$ and subsequently freeze dried (Freeze Dry System, Labconco Corporation, Kansas City, MO). Lyophilized constructs were digested using a Proteinase $\mathrm{K}$ solution (Sigma) for 16 to $20 \mathrm{~h}$ at $55^{\circ} \mathrm{C}$, and digested samples were frozen at $-20^{\circ} \mathrm{C}$ for at least $24 \mathrm{~h}$ to help ensure cell lysis, because CyQuant GR is a membrane-impermeable DNA stain. Fifty $\mu \mathrm{L}$ of each sample was then combined with $150 \mu \mathrm{L}$ of CyQuant GR dye solution in $1 X$ cell lysis buffer to yield a final dye concentration of $1 X$. Samples were loaded into a 96-well plate along with cell standards $\left(6.25 \times 10^{4}-1.00 \times 10^{6}\right.$ cells $\left./ \mathrm{mL}\right)$ and DNA standards $(2-30 \mu \mathrm{g} / \mathrm{mL})$, incubated in the dark for $5 \mathrm{~min}$, and read us- ing a fluorescence plate reader (Bio-Tek, Winooski, VT) set at 485-nm excitation and 528-nm emission. Fluorescence intensity was correlated to DNA and cell standards to estimate the number of cells within each construct.

\section{Cell morphology}

Cell morphology was assessed qualitatively and quantitatively at days 3,7 , and 14 . Samples were fixed and permeabilized in $0.1 \%$ Triton X-100 (Sigma). The actin network was visualized using Alexa Fluor 488-conjugated Phalloidin (Molecular Probes) diluted 1:100 in 1\% bovine serum albumin (Sigma) for $30 \mathrm{~min}$. Nuclei were stained with EH (Molecular Probes) diluted 1:500 in PBS for $20 \mathrm{~min}$. Constructs were washed twice and visualized on a laser scanning confocal microscope (Carl Zeiss) using 488-nm and 543-nm lasers at $20 \times$ magnification. Z-stacks ranging from 50 - to $90-\mu \mathrm{m}$ thick were taken of the interior of each construct, again imaging both sides and keeping z-stack locations consistent for all conditions.

\section{Image analysis}

Neurolucida software (MBF Biosciences, Williston, VT) was used to identify individual nuclei and to trace actin in 3D space. Preliminarily, to correlate actin to cell morphology, low-density SCs on tissue culture-treated glass coverslips were stained and imaged for actin. Additional phase microscopy images were taken at the same locations to visualize cell morphology. Tracings of actin networks via Neurolucida (described below) and tracings of the whole cell were then plotted to compare size and shape, demonstrating that actin networks were an accurate estimation of cell morphology ( 0.92 correlation factor). This step was necessary because it is much easier to visualize actin in $3 \mathrm{D}$ constructs than it is to visualize cell membrane boundaries.

In 3D constructs, Neurolucida traces were used to quantify the length and number of branches of the actin networks $(n=3$ replicates of each construct type-at least 6 image stacks for each replicate were analyzed). Any partial cells were disregarded so that only cells that could be traced in their entirety were analyzed. Although much of the Neurolucida tracing is automated, some user intervention was required when two actin networks were intimately touching one another. In this case, actin tracings were manually separated at a distance halfway between the two nuclei containing each of the actin networks, which underestimates the absolute length because it is likely that the tracings overlap one another. Even though the absolute length could not be accurately ascertained, because this convention was upheld over the course of all tracings and construct types, relative measurements were valid. Neurolucida Explorer was used to automatically analyze the average actin process length and number of processes for each full cell within an image stack; in this work, a cell process was defined as a protrusion from the SC.

\section{Statistics}

One-way analysis of variance was used to determine whether any significant differences existed between groups in a data set, and Tukey's multiple comparisons test was subsequently used to evaluate all pairwise comparisons. Significance was set at $p<0.05$. 

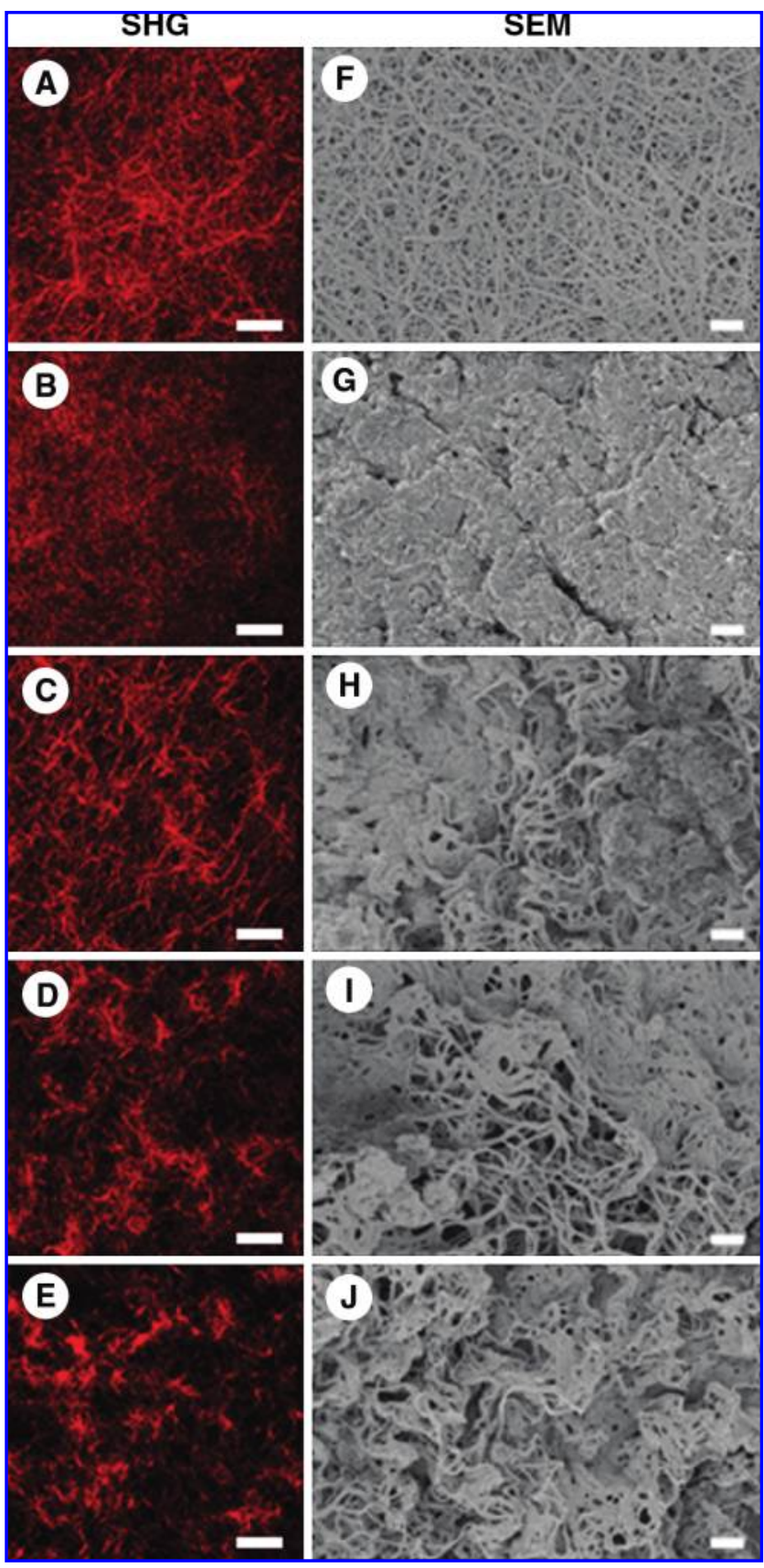

FIG. 1. Second harmonic generation images of the collagen I network within (A) collagen-only, (B) 10\% Matrigel, (C) 20\% Matrigel, (D) 35\% Matrigel, and (E) 50\% Matrigel constructs. Collagen I autofluorescence is shown in red and is distributed nonuniformly in the higher-percentage Matrigel constructs, resulting in regions with greater relative concentrations of collagen I or Matrigel proteins. Images are two-dimensional projections of $15-\mu \mathrm{m}$-thick z-stacks. Scale bars: $20 \mu \mathrm{m}, 63 \times$ magnification. Scanning electronic microscopy images of (F) collagen-only, (G) 10\% Matrigel, (H) 20\% Matrigel, (I) 35\% Matrigel, and (J) 50\% Matrigel constructs. Scale bars: $1 \mu \mathrm{m}, 25,000 \times$ magnification. Color images available online at www.liebertonline.com/ten.

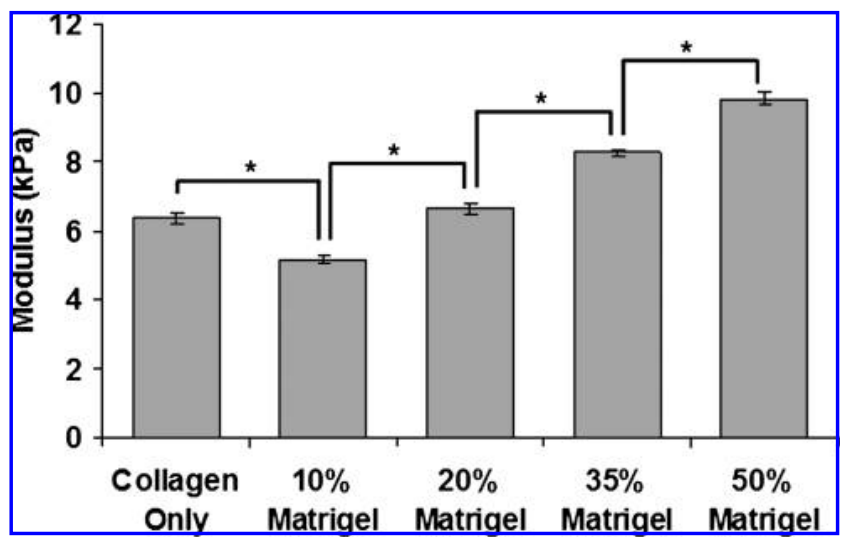

FIG. 2. Young's moduli of the various collagen-Matrigel compositions. Moduli were obtained through displacement control unconfined compression at a rate of $0.5 \mathrm{~mm} / \mathrm{min}$. Moduli were calculated from the linear region of the stressstrain curve, which was assumed to be between $15 \%$ and $50 \%$ of the maximal stress. After an initial drop in elastic moduli, constructs became increasingly stiffer with the addition of Matrigel. Bars are means \pm standard errors. *Significant statistical differences $(p<0.05) . \mathrm{n}=3$ separate trials.

\section{Results}

\section{Matrix composition}

SHG imaging revealed varied collagen I distribution within the different construct types (Fig. 1A-E). Collagen I autofluorescence is shown in red, and a dense network of uniformly distributed fibrils, which is a triple helix of collagen I protein chains, can be seen within the $100 \%$ collagen constructs (Fig. 1A). This dense network was notably absent as the percentage of Matrigel increased to 35\% and 50\% (Fig. 1D, E). In these constructs, the collagen I appeared less interconnected and less uniformly distributed throughout, forming distinct islands of higher collagen concentrations amidst the Matrigel. Ten percent (Fig. 1B) and 20\% (Fig. 1C) Matrigel constructs showed a progression from the dense network of fibrils seen in the collagen-only constructs to the clustering of collagen I present in the $35 \%$ and $50 \%$ Matrigel conditions.

SEM images also illustrated differences in matrix structure. Again, in the collagen-only matrix, a dense network of highly interconnected collagen I fibrils could be visualized (Fig, 1F). Upon incorporation of $10 \%$ Matrigel (Fig. 1G), this welldefined network disappeared, replaced by tightly packed fibrils with fewer and smaller pores. As the percentage of Matrigel increased from $20 \%$ to $35 \%$ to $50 \%$ (Fig. $1 \mathrm{H}$ to J, respectively) fibrils and pores again began to appear within the matrices, although these fibrillar networks looked distinctly different from those seen in the collagen-only constructs. SEM imaging, however, cannot definitively identify collagen I fibrils in a Matrigel matrix. Fibrils here formed a less-interconnected network and appeared more tortuous in nature. Additionally, regions of fibrils containing relatively large pores could be seen adjacent to areas of tightly packed matrix, reminiscent of that seen in the $10 \%$ Matrigel constructs. Accurate quantification of pore size was difficult in these samples because of changes in construct volume during the drying process, but becase this effect was constant over all conditions, relative comparisons were valid. 


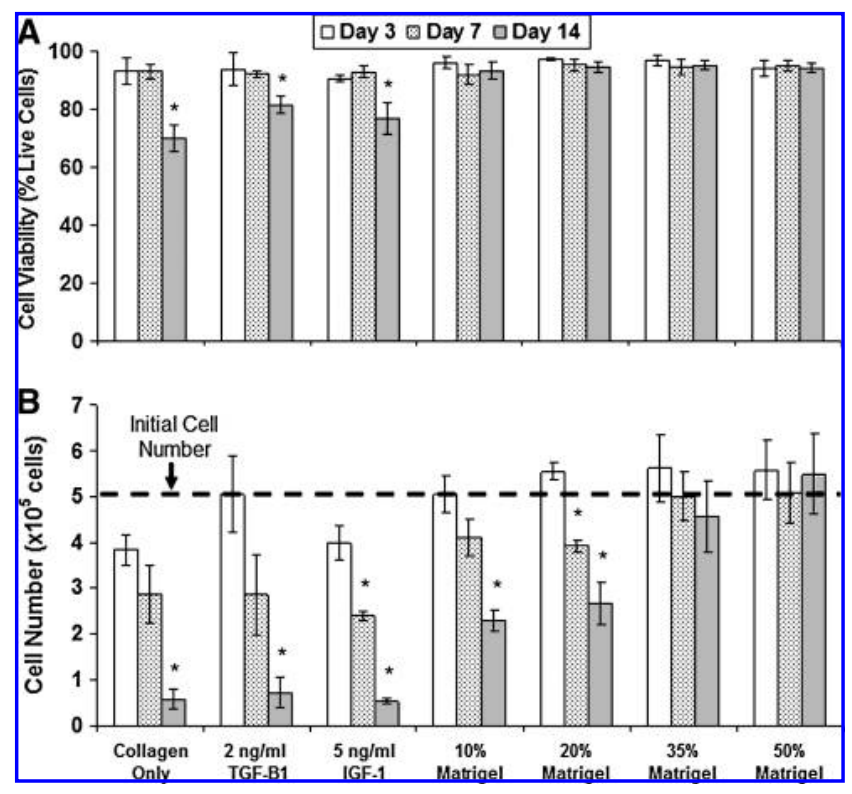

FIG. 3. (A) Cell viability on days 3, 7, and 14 in culture, displayed as the percentage of living cells. Live and dead cells were stained using calcein and ethidium homodimer, respectively, and analyzed using FARSIGHT software. Cell viability was greater than $90 \%$ for all constructs on days 3 and 7. On day 14, only constructs containing Matrigel had viabilities greater than $90 \%$. (B) Cell numbers assessed at days 3 , 7 , and 14 via a DNA assay. Initial seeding density was $5 \times 10^{5}$ cells/construct, and after 14 days in culture, increasing percentages of Matrigel correlated to higher cell numbers. Fifty percent Matrigel constructs supported the highest number of cells at 14 days, with $5.4 \times 10^{5}$ cells/construct, whereas collagen-only constructs averaged $5.8 \times 10^{4}$ cells, an $88 \%$ decrease from the initial cell seeding number. Bars are means \pm standard errors. *Significant decrease from the day 3 value for each condition $(p<0.05) . \mathrm{n}=3$ trials for viability data and $\mathrm{n}=4$ for cell number data.

\section{Compliance testing}

Acellular constructs were subjected to compression testing and exhibited differences in mechanical properties. The stiffest construct was the 50\% Matrigel, exhibiting an elastic modulus of $9.8 \mathrm{kPa}$ (Fig. 2). A reduction in Matrigel resulted in a linear decline in modulus, with the $10 \%$ Matrigel being most compliant at $5.1 \mathrm{kPa}$. The collagen-only construct had a slightly higher modulus of $6.4 \mathrm{kPa}$. Statistical differences were detected between all groups $(p<0.001)$ except between the $20 \%$ Matrigel and collagen-only constructs, because these conditions had similar moduli $(6.6 \mathrm{kPa}$ and $6.4 \mathrm{kPa}$, respectively).

\section{Cell viability}

Cell viability was measured using vital cell dye and expressed as the percentage of living cells on days 3,7 , and 14 (Fig. 3A). Viability was greater than $90 \%$ for all construct types on days 3 and 7, and no differences were detected between any of these groups. By day 14, SC viability within the collagen-only, $2 \mathrm{ng} / \mathrm{mL}-\mathrm{TGF}-\beta 1$, and $5 \mathrm{ng} / \mathrm{mL}-\mathrm{IGF}-1$ constructs dropped to $70 \%, 81 \%$, and $77 \%$, respectively. Any constructs containing Matrigel retained viability values of greater than $93 \%$ on day 14 , significantly higher than those of the collagen-only constructs with and without added growth factors $(p<0.05)$. The vital cell dye was functional because dead cells were observed in all samples. Viability was constant regardless of location within the construct.

\section{Cell number}

Cell number in constructs was estimated according to total DNA using the CyQuant GR DNA assay on days 3, 7, and 14 (Fig. 3B). Fivex $10^{5}$ cells were incorporated into each construct on day 0 , and this number was not exceeded on any day. No significant differences between groups were detected on day 3. By day $7,35 \%$ and $50 \%$ Matrigel constructs has significantly more cells than collagen constructs with and without growth factors; no differences were detected between Matrigel-containing constructs on day 7. By day 14, the increase in cell number correlated to the increase in Matrigel percentage was much more pronounced. The 35\% and 50\% Matrigel constructs had significantly greater cell numbers than any other constructs $(p<0.05)$. After 14 days in culture, although cell number was highest in the 50\% Matrigel construct, no significant difference in number was detected between the 35\% and 50\% Matrigel conditions and the day 3 values; all other constructs had significantly fewer cells at day 14 than at day $3(p<0.05)$. Neither IGF-1 nor TGF- $\beta 1$ had greater cell numbers than collagen-only controls over 14 days in culture.

\section{Cell morphology}

Cell morphology was examined via actin networks on days 3,7 , and 14 for all construct types. Actin networks, shown in Figure 4 as 2D projections of 3D image stacks, were traced and analyzed as an indicator of cell morphology. Preliminarily, SCs were cultured on glass coverslips and whole-cell spreading (whole-cell tracing in phase micrographs) was correlated ( 0.92 correlation factor) to the tracing of actin in fluorescence micrographs, indicating that actin was an accurate estimation of SC size and shape (data not shown).

Within the collagen-only constructs, a spherical SC morphology was observed (Fig. 4A) and the addition of neither TGF- $\beta 1$ nor IGF-1 elicited dramatic changes in morphology, although a few small processes can be seen protruding from these cells (Fig. 4B and C, respectively), resulting in 1.4 times and 1.2 times longer average process lengths, respectively, than with collagen-only controls (Fig. 5A). However, the incorporation of Matrigel resulted in significant changes in cell morphology. All constructs containing Matrigel had significantly longer average process lengths $(p<0.001)$, with the $10 \%, 20 \%, 35 \%$, and $50 \%$ constructs achieving $2.4,3.5,3.7$, and 4.2 times longer average process length than with collagenonly controls (Fig. 5A). Even at 10\% Matrigel, numerous thin processes extended from the cell body (Fig. 4D), with an average of 3.8 processes per cell. Although these processes were significantly shorter, more processes per cell were present than under other condition $(p<0.001)$. As the percentage of Matrigel was increased from $20 \%$ to $35 \%$ to $50 \%$ (Fig. 4E to G, respectively), processes became noticeably longer and straighter, with fewer processes present on each cell within the higher-percentage Matrigel constructs (Fig. 5B). Despite this, no significant changes in number of processes per cell were detected between these groups, and only the $20 \%$ and $50 \%$ Matrigel constructs differed significantly from each other 

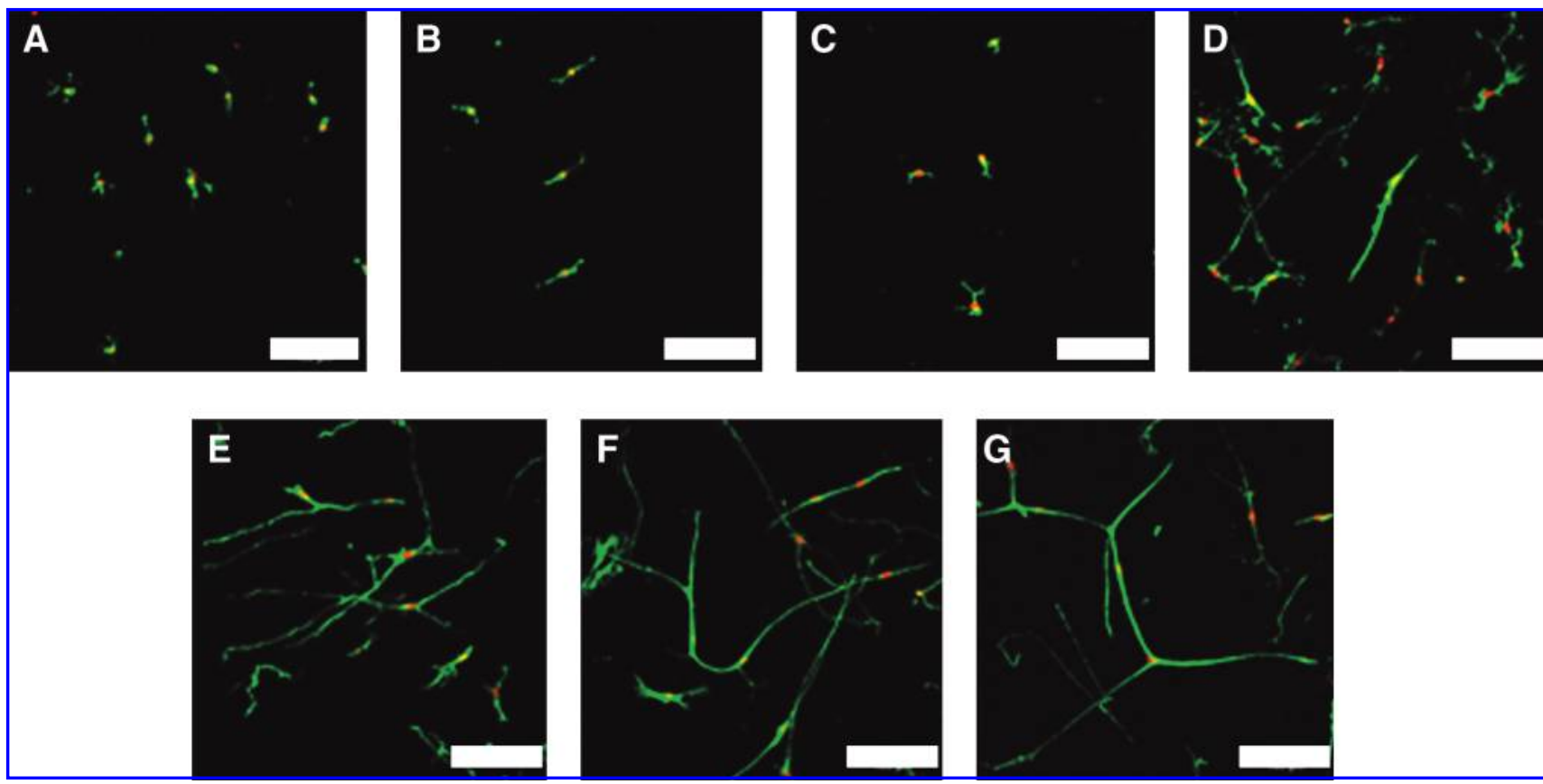

FIG. 4. Schwann cell morphology within the various constructs. Cells within the (A) collagen-only constructs were spherical in morphology, and those in the (B) collagen-only constructs with $2 \mathrm{ng} / \mathrm{mL}$ of transforming growth factor beta 1 or $(\mathbf{C}) 5 \mathrm{ng} / \mathrm{mL}$ of insulinlike growth factor-1 extended a limited number of short processes. Thin but longer processes appeared upon the incorporation of (D) 10\% and (E) 20\% Matrigel. These processes became visibly longer and straighter as the Matrigel percentage was increased to (F) 35\% and (G) 50\%. Actin was stained green using Alexa-Fluor 488-conjugated phalloidin, and nuclei were stained red using ethidium homodimer. Images are two-dimensional projections of $40-\mu \mathrm{m} z$-stacks obtained using a confocal microscope. Scale bars: $100 \mu \mathrm{m}, 20 \times$ magnification. Color images available online at www.liebertonline.com/ten.

with respect to average process length $(p<0.001)$. Collagenonly constructs and collagen constructs with TGF- $\beta 1$ or IGF-1 contained an average of 2.4, 2.2, and 2.3 small processes per cell (Fig. 5B), respectively, and these differed significantly from the $10 \%$ Matrigel condition only ( $p<0.001)$. Data for days 3, 7 , and 14 were pooled together for each construct type, because no differences in morphology were detected between days, indicating that cell spreading occurred within 3 days in culture. Additionally, cell morphology was consistent throughout the volume of each construct. Over 14 days, processes were longest in the 50\% Matrigel constructs, although differences from the $35 \%$ Matrigel condition were not significant.

\section{Discussion}

This study lays a foundation for our long-term goal of achieving directed neuronal regeneration. Scaffolds for nerve repair are typically optimized for neurite growth, whereas the glial response to the materials is less well studied. In this study, we have taken an alternate approach by creating materials that additionally support SCs. As discussed, SCs possess the ability to support and can direct neurite growth, and our overall aim is to use oriented SCs in a 3D in vitro matrix environment to promote and guide neurite extension. Achieving this effect requires that SCs interact with the matrix such that they survive and elongate. By using 3D collagenMatrigel composite scaffolds, we have created mechanically robust materials that allow SC extension and therefore may have utility in directing neurite outgrowth.

SHG imaging revealed the distinct structural differences between the various matrix types examined in this study and portrayed the distribution of collagen I amid the Matrigel. Collagen I has a triple-helix structure and a high degree of crystallinity, making it visible to $\mathrm{SHG} .{ }^{28}$ Additionally, strong chiroptical properties enhance its autofluorescence. These chiroptical properties are not present in collagen IV or laminin, and therefore these proteins are not visualized using SHG imaging. ${ }^{30}$ Brown et al. reported no SHG activity from Matrigel proteins using SHG settings similar to those in this study. ${ }^{31}$ Therefore, we are confident that the autofluorescence viewed in our samples was a result of the fibrillar collagen I distribution.

Collagen I is a mechanically robust structural protein, and its distribution affects the mechanical structure and resulting strength of the bulk material. In vitro, collagen constructs are easily handled, which is of practical importance when contemplating their use as engineered tissues in the clinic. In addition, previous work has demonstrated that the collagen fibrils within constructs can be directionally aligned using a variety of techniques. ${ }^{32,33}$ Matrix alignment offers the possibility of creating SC outgrowth in prescribed directions and will also promote associated aligned neurite outgrowth. For these reasons, collagen I was used as the primary matrix material for the constructs in this study.

Incorporation of Matrigel caused distinct changes in the mechanical properties and structure of the constructs while also altering the cellular response. Addition of Matrigel resulted in more-fragile constructs, although all compositions used in this study were robust enough to be handled without damage. Constructs containing more than 50\% Matrigel could not be practically handled without tearing and were therefore not investigated further. Constructs with higher Matrigel 


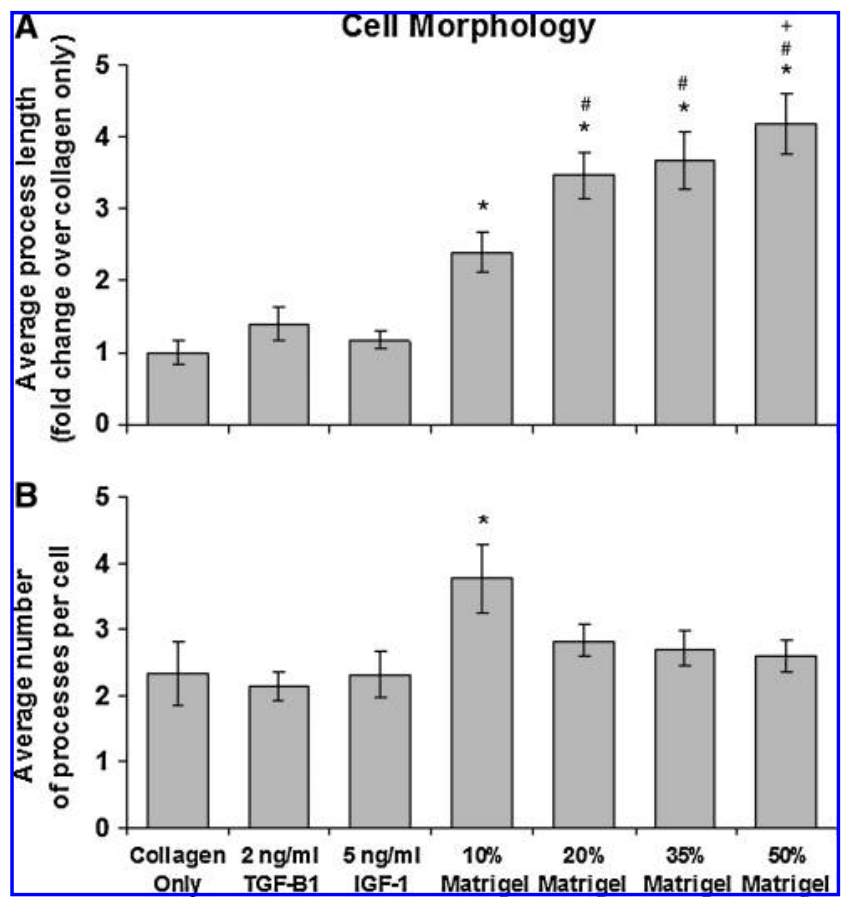

FIG. 5. (A) Average process length and (B) average number of processes per cell. Actin networks stained using Alexa fluor 488-conjugated phalloidin were traced and analyzed using Neurolucida software. Values were averaged over 14 days of culture for each construct type. Process length increased with the percentage of Matrigel, reaching 4.2 times as great as the collagen-only condition in the $50 \%$ Matrigel case. The number of processes was significantly greater only in the $10 \%$ Matrigel case, in which there was an average of 3.8 processes/cell. Bars are means \pm standard errors. Significantly greater than ${ }^{*}$ collagen control, ${ }^{\#} 10 \%$, Matrigel, and ${ }^{+} 20 \%$ Matrigel $(p<0.05)$. $\mathrm{n}=3$ separate trials with six images stacks being analyzed for each condition in each trial ( $\sim 10$ cells/image stack).

content may have ripped more easily because the highly interconnected collagen fibril network seen in pure-collagen constructs was disrupted, replaced by islands of collagen (Fig. 1E). At low Matrigel concentrations (e.g., 10\%, Fig. 1G), the matrix structure as observed using SEM was uniform, with a fine fiber structure, whereas at higher percentages of Matrigel, larger fibrils reappeared (Fig. 1H-J). It is likely that the observed fibrils are collagen domains amid more-compact Matrigel matrix, although SEM imaging does not allow us to reliably distinguish between these proteins. The observed changes in fibrillar structure are also supported by Figure 2; the addition of Matrigel initially resulted in a lower modulus than in collagen controls, yet further addition of Matrigel ultimately yielded constructs with moduli that exceeded those of collagen controls. Additionally, SHG images (Fig. 1, which image only the fibrillar collagen structure) support the SEM observations, showing domains of collagen I at higher Matrigel concentrations. The incorporation of Matrigel altered the mechanical and architectural properties of the matrices, although the mechanism of this effect requires further study.

Compliance of a substrate directly affects the phenotype of cells that are in contact with it. ${ }^{34,35}$ Previous research has shown the importance of mechanical stiffness in neural tissue engineering; for instance, Leach et al. demonstrated that there was less branching of PC12 neurites on compliant materials below a certain threshold. ${ }^{36}$ Much of the published work, however, has involved quantifying neurite outgrowth, rather than glial cell morphology. ${ }^{37,38}$ In our study, there was a direct correlation between greater stiffness and SC spreading, with the stiffer constructs inducing a greater degree of spreading. Nevertheless, we were not able to discriminate between the effects of the mechanical properties and the biological effects of the protein components in the Matrigel. The pure-collagen samples had moduli that were not significantly different from those of the $20 \%$ Matrigel samples, yet SC spreading was different between these constructs (Fig. 4A, E). Even though the $10 \%$ Matrigel samples were significantly more compliant than the pure collagen constructs, greater spreading was still observed in the presence of Matrigel. These results suggest that matrix compliance is not the only factor affecting SC extension and that the biological cues from Matrigel also contribute.

Cell viability in all constructs was high, although there were significant drops at day 14 of culture in the pure collagen and collagen with TGF- $\beta 1$ or IGF-1. Cell number in the 3D constructs tended to increase with increasing Matrigel content, especially at the later time points. For anchoragedependent cell types, inhibition of cell spreading has been shown to trigger apoptosis. ${ }^{39}$ Chen et al. reported that cell shape governed whether individual cells grew or died and that when cell shape and spreading were sufficiently restricted, apoptosis was induced. ${ }^{40}$ Additionally, Nakao et al. reported that more than $50 \%$ of harvested SCs undergo apoptosis after losing contact with axons but that these SCs could be rescued from apoptosis by increasing their adhesion using different substrata. ${ }^{41}$ These results suggest that a lack of cell adherence to the matrix, and the associated lack of cell spreading, was responsible for cell death in the constructs with lower Matrigel content. Viability data showed that the cells that remained in the constructs were viable, although the cell number data demonstrate that there were fewer of them as the amount of Matrigel decreased. Because viability data are only a snapshot in time, it is likely that dead cells degraded over time and that therefore only viable cells remained at longer time points. Our results show that the 35\% and 50\% Matrigel scaffolds were most supportive of cell maintenance over 14 days in culture.

The main extracellular matrix proteins in the nervous system in contact with SC-axon units are the basal lamina proteins laminin and collagen IV. Collagen I is not a major protein in contact with these cells because it lies outside the basal lamina in the endoneurium. ${ }^{25}$ Therefore, it is likely that the addition of Matrigel allowed SCs to preferentially attach to the proteins of their native environment and extend processes in the composite matrices used in this study. Addition of Matrigel to a construct necessarily results in a corresponding decrease in collagen I content (because all constructs were made at the same total protein content). However, we verified that SC extension was independent of the collagen I concentration by creating controls with variable collagen I content $(1.0-2.0 \mathrm{mg} / \mathrm{mL}$ of collagen) in the absence of Matrigel. These collagen concentrations corresponded to the concentrations achieved by the addition of the various levels of Matrigel in this study. Regardless of collagen I concentration, SCs remained in a spherical morphology in the absence of Matrigel. Additionally, we did not achieve any increase in 
SC survival by lowering the collagen I concentration. Matrix compliance may have played a role in determining the degree of SC extension, but our overall our data suggest that the addition of Matrigel, and not the decrease in collagen I, was responsible for cell spreading.

Addition of exogenous TGF- $\beta 1$ at $2 \mathrm{ng} / \mathrm{mL}$ or IGF- 1 at $5 \mathrm{ng} / \mathrm{mL}$ to collagen I constructs did not elicit cell spreading at a level comparable with that achieved by adding Matrigel. It has been reported that TGF- $\beta 1$ causes SCs to spread within collagen I matrices at concentrations as low as $0.5 \mathrm{ng} / \mathrm{mL}^{19}$ although this work classified cells as spread or unspread based on the presence or absence of cellular processes, respectively, and consequently expressed cell spreading as the percentage of cells classified as containing processes. In the current work, we quantified the length of processes and not the percentage of cells adopting a spread morphology, making direct comparisons difficult. We did, however, observe a 1.4 times greater average process length with the addition TGF- $\beta 1$ than in collagen-only controls (Fig. 5A), and small processes could be seen protruding from many of the cells in TGF- $\beta 1$-containing constructs (Fig. 4B). Therefore, TGF- $\beta 1$ at $2 \mathrm{ng} / \mathrm{mL}$ had some effect in changing cell morphology, although the addition of Matrigel resulted in substantial changes. TGF- $\beta 1$ and IGF- 1 are the only growth factors that are not effectively eliminated in the GFR formulation of Matrigel and are present at concentrations of approximately $1.7 \mathrm{ng} / \mathrm{mL}$ and $5 \mathrm{ng} / \mathrm{mL}$, respectively. ${ }^{24}$ Because we observed little increase in cell spreading with the exogenous addition of either of these growth factors to collagen I constructs, it is likely that process extension is occurring because of the presence of laminin and collagen IV in the Matrigel mixture, proteins typically found in the basal lamina.

These results show that the relative amounts of collagen Type I and Matrigel in composite scaffolds in vitro alter SC morphology and behavior in a $3 \mathrm{D}$ matrix. Collagen I and Matrigel both have desirable properties for use in creating engineered neural tissues; collagen I is a mechanically robust protein that can be directionally aligned, whereas Matrigel can provide a suitable environment for SC adhesion and extension. Additionally, each material has been used previously in the PNS and CNS in vivo and has been shown to be permissive to neurite extension, ${ }^{11,13,23,42}$ although use of these proteins separately is not suitable in creating a neural guidance scaffold because SC spreading and growth are not observed in pure-collagen constructs, whereas pure-Matrigel constructs are mechanically fragile and do not contain robust fibers for alignment. The composite constructs we studied retained positive properties of each component, although these properties changed with the relative concentrations of the protein components. In future work, we will use these composite protein scaffolds in conjunction with scaffold alignment, soluble factors, and external forces to promote and direct the extension of neurites and migration of SCs through the scaffold biomaterial. The potential synergy of multiple cues presented to the supporting SCs and regenerating axons may lead to a clinically effective scaffold for injuries to the nervous system.

\section{Acknowledgments}

The authors would like to thank Dr. Chris Bjornsson and Megan Farrell for their technical assistance. This work was supported by the New York State Spinal Cord Injury Research Program IDEA \#J50480 (DMT and JPS) and completed in the Center for Biotechnology and Interdisciplinary Studies at Rensselaer Polytechnic Institute.

\section{Disclosure Statement}

No competing financial interests exist.

\section{References}

1. Khan, Y., and Rajadhyaksha, M.S. Glial cells: The other cells of the nervous system. Resonance 7, 8, 2002.

2. Salzer, J.L. Clustering sodium channels at the node of Ranvier: close encounters of the axon-glia kind. Neuron 18, 843, 1997.

3. Bunge, R.P. The role of the Schwann cell in trophic support and regeneration. J Neurol 242, 19, 1994.

4. Carey, D.J., Eldridge, C.F., Cornbrooks, C.J., Timpl, R., and Bunge, R.P. Biosynthesis of type IV collagen by cultured rat Schwann cells. J Cell Biol 97, 473, 1983.

5. Cornbrooks, C.J., Carey, D.J., McDonald, J.A., Timpl, R., and Bunge, R.P. In vivo and in vitro observations on laminin production by Schwann cells. Proc Natl Acad Sci U S A 80, 3850, 1983.

6. Williams, L.R., Longo, F.M., Powell, H.C., Lundborg, G., and Varon, S. Spatial-temporal progress of peripheral nerve regeneration within a silicone chamber: parameters for a bioassay. J Comp Neurol 218, 460, 1983.

7. Hall, S.M. The Schwann cell: a reappraisal of its role in the peripheral nervous system. Neuropathol Appl Neurobiol 4, 165, 1978.

8. Miller, C., Jeftinija, S., and Mallapragada, S. Micropatterned Schwann cell-seeded biodegradable polymer substrates significantly enhance neurite alignment and outgrowth. Tissue Eng 7, 705, 2001.

9. Thompson, D.M., and Buettner, H.M. Oriented Schwann cell monolayers for directed neurite outgrowth. Ann Biomed Eng 32, 1120, 2004.

10. Rodriguez, F.J., Verdu, E., Ceballos, D., and Navarro, X. Nerve guides seeded with autologous Schwann cells improve nerve regeneration. Exp Neurol 161, 571, 2000.

11. Guenard, V., Kleitman, N., Morrissey, T.K., Bunge, R.P., and Aebischer, P. Syngeneic Schwann cells derived from adult nerves seeded in semipermeable guidance channels enhance peripheral nerve regeneration. J Neurosci 12, 3310, 1992.

12. Noble, M., Fok-Seang, J., and Cohen, J. Glia are a unique substrate for the in vitro growth of central nervous system neurons. J Neurosci 4, 1892, 1984.

13. Xu, X.M., Guenard, V., Kleitman, N., and Bunge, M.B. Axonal regeneration into Schwann cell-seeded guidance channels grafted into transected adult rat spinal cord. J Comp Neurol 351, 145, 1995.

14. Xu, X.M., Chen, A., Guénard, V., Kleitman, N., and Bunge, M.B. Bridging Schwann cell transplants promote axonal regeneration from both the rostral and caudal stumps of transected adult rat spinal cord. J Neurocytol 26, 1, 1997.

15. Xu, X.M., Zhang, S.X., Li, H., Aebischer, P., and Bunge, M.B. Regrowth of axons into the distal spinal cord through a Schwann-cell-seeded mini-channel implanted into hemisected adult rat spinal cord. Eur J Neurosci 11, 1723, 1999.

16. Oudega, M., and Xu, X.M. Schwann cell transplantation for repair of the adult spinal cord. J Neurotrauma 23, 453, 2006.

17. Bachelin, C., Lachapelle, F., Girard, C., Moissonnier, P., Serguera-Lagache, C., Mallet, J., Fontaine, D., Chojnowski, 
A., Le Guern, E., Nait-Oumesmar, B., and Baron-Van Evercooren, A. Efficient myelin repair in the macaque spinal cord by autologous grafts of Schwann cells. Brain 128, 540, 2005.

18. Hurtado, A., Moon, L.D., Maquet, V., Blits, B., Jerome, R., and Oudega, M. Poly (D,L-lactic acid) macroporous guidance scaffolds seeded with Schwann cells genetically modified to secrete a bi-functional neurotrophin implanted in the completely transected adult rat thoracic spinal cord. Biomaterials 27, 430, 2006.

19. Rosner, B.I., Hang, T., and Tranquillo, R.T. Schwann cell behavior in three-dimensional collagen gels: Evidence for differential mechano-transduction and the influence of TGFbeta 1 in morphological polarization and differentiation. Exp Neurol 195, 81, 2005.

20. Dubey, N., Letourneau, P.C., and Tranquillo, R.T. Guided neurite elongation and Schwann cell invasion into magnetically aligned collagen in simulated peripheral nerve regeneration. Exp Neurol 158, 338, 1999.

21. Phillips, J.B., Bunting, S.C., Hall, S.M., and Brown, R.A. Neural tissue engineering: a self-organizing collagen guidance conduit. Tissue Eng 11, 1611, 2005.

22. Chen, Y.S., Hsieh, C.L., Tsai, C.C., Chen, T.H., Cheng, W.C., $\mathrm{Hu}, \mathrm{C} . \mathrm{L}$. , and $\mathrm{Yao}, \mathrm{C} . \mathrm{H}$. Peripheral nerve regeneration using silicone rubber chambers filled with collagen, laminin and fibronectin. Biomaterials 21, 1541, 2000.

23. Ceballos, D., Navarro, X., Dubey, N., Wendelschafer-Crabb, G., Kennedy, W.R., and Tranquillo, R.T. Magnetically aligned collagen gel filling a collagen nerve guide improves peripheral nerve regeneration. Exp Neurol 158, 290, 1999.

24. BD Biosciences. BD Matrigel Basement Membrane Matrix. 2007 [on-line]. Available at http://www.bdbiosciences.com/ discovery_labware/products/display_product.php?keyID= 230 accessed on July 15, 2008.

25. Chernousov, M.A., and Carey, D.J. Schwann cell extracellular matrix molecules and their receptors. Histol Histopathol 15, 593, 2000.

26. Einheber, S., Hannocks, M.J., Metz, C.N., Rifkin, D.B., and Salzer, J.L. Transforming growth factor-beta 1 regulates axon/Schwann cell interactions. J Cell Biol 129, 443, 1995.

27. Stephens, D.J., and Allan, V.J. Light microscopy techniques for live cell imaging. Science 300, 82, 2003.

28. Cox, G., Kable, E., Jones, A., Fraser, I., Manconi, F., and Gorrell, M.D. 3-Dimensional imaging of collagen using second harmonic generation. J Struct Biol 141, 53, 2003.

29. Bjornsson, C.S., Lin, G., Al-Kofahi, Y., Narayanaswamy, A., Smith, K.L., Shain, W., and Roysam, B. Associative image analysis: a method for automated quantification of 3D multiparameter images of brain tissue. J Neurosci Methods 170, 165, 2008.

30. Pena, A.M., Boulesteix, T., Dartigalongue, T., and SchanneKlein, M.C. Chiroptical effects in the second harmonic signal of collagens I and IV. J Am Chem Soc 127, 10314, 2005.

31. Brown, E., and McKee, T. Dynamic imaging of collagen and its modulation in tumors in vivo using second-harmonic generation. Nat Med 9, 796, 2003.
32. Barocas, V.H., Girton, T.S., and Tranquillo, R.T. Engineered alignment in media equivalents: magnetic prealignment and mandrel compaction. J Biomech Eng 120, 660, 1998.

33. Costa, K.D., Lee, E.J., and Holmes, J.W. Creating alignment and anisotropy in engineered heart tissue: role of boundary conditions in a model three-dimensional culture system. Tissue Eng 9, 567, 2003.

34. Yeung, T., Georges, P.C., Flanagan, L.A., Marg, B., Ortiz, M., Funaki, M., Zahir, N., Ming, W., Weaver, V., and Janmey, P.A. Effects of substrate stiffness on cell morphology, cytoskeletal structure, and adhesion. Cell Motil Cytoskeleton 60, 24, 2005.

35. Engler, A.J., Sen, S., Sweeney, H.L., and Discher, D.E. Matrix elasticity directs stem cell lineage specification. Cell 126, 677, 2006.

36. Leach, J.B., Brown, X.Q., Jacot, J.G., Dimilla, P.A., and Wong, J.Y. Neurite outgrowth and branching of PC12 cells on very soft substrates sharply decreases below a threshold of substrate rigidity. J Neural Eng 4, 26, 2007.

37. Georges, P.C., Miller, W.J., Meaney, D.F., Sawyer, E.S., and Janmey, P.A. Matrices with compliance comparable to that of brain tissue select neuronal over glial growth in mixed cortical cultures. Biophys J 90, 3012, 2006.

38. Balgude, A.P., Yu, X., Szymanski, A., and Bellamkonda, R.V. Agarose gel stiffness determines rate of DRG neurite extension in 3D cultures. Biomaterials 22, 1077, 2001.

39. Re, F., Zanetti, A., Sironi, M., Polentarutti, N., Lanfrancone, L., Dejana, E., and Colotta, F. Inhibition of anchoragedependent cell spreading triggers apoptosis in cultured human endothelial cells. J Cell Biol 127, 537, 1994.

40. Chen, C.S., Mrksich, M., Huang, S., Whitesides, G.M., and Ingber, D.E. Geometric control of cell life and death. Science 276, 1425, 1997.

41. Nakao, J., Shinoda, J., Nakai, Y., Murase, S., and Uyemura, K. Apoptosis regulates the number of Schwann cells at the premyelinating stage. J Neurochem 68, 1853, 1997.

42. Houweling, D.A., Lankhorst, A.J., Gispen, W.H., Bar, P.R., and Joosten, E.A. Collagen containing neurotrophin-3 (NT3) attracts regrowing injured corticospinal axons in the adult rat spinal cord and promotes partial functional recovery. Exp Neurol 153, 49, 1998.

Address correspondence to: Jan P. Stegemann, Ph.D. Associate Professor Department of Biomedical Engineering 1101 Beal Ave. University of Michigan Ann Arbor, MI 48109

E-mail: jpsteg@umich.edu

Received: July 16, 2008 Accepted: February 19, 2009

Online Publication Date: June 30, 2009 

This article has been cited by:

1. Muthulekha Swamydas, Jill M. Eddy, Karen J. L. Burg, Didier Dréau. 2010. Matrix compositions and the development of breast acini and ducts in 3D cultures. In Vitro Cellular \& Developmental Biology - Animal 46:8, 673-684. [CrossRef]

2. Jerani T. S. Pettikiriarachchi, Clare L. Parish, Molly S. Shoichet, John S. Forsythe, David R. Nisbet. 2010. Biomaterials for Brain Tissue Engineering. Australian Journal of Chemistry 63:8, 1143. [CrossRef] 